Check for updates

Cite this: RSC Adv., 2019, 9, 15196

Received 14th January 2019

Accepted 5th May 2019

DOI: $10.1039 / c 9 r a 00330 d$

rsc.li/rsc-advances

\section{Hepatitis B virus S gene therapy with $10-23$ DNAzyme delivered by chitosan-g-stearic acid micelles}

\author{
Yun Hong, ${ }^{a}$ Dongsen Mao, ${ }^{b}$ Rui Wu, ${ }^{c}$ Zhe Gao, ${ }^{a}$ Tingting Meng, ${ }^{c}$ Rongrong Wang, ${ }^{a}$ \\ Lin $\mathrm{Liu}^{\mathrm{a}}$ and Jing Miao (D)*a
}

DNAzymes have the potential to suppress gene expression through sequence-specific mRNA cleavage and can therefore play an important role in various gene therapies. Hepatitis B virus (HBV) is still one of the most serious liver infections in people around the world and is difficult to treat. We previously designed a 10-23 DNAzyme called DrzBS, which targets HBV S gene expression, but this enzyme depends on exogenous delivery, and so its application has been limited. To overcome this limitation, we have now developed a chitosan-based nanocarrier (chitosan-g-stearic acid, CSO-SA) for intracellular delivery of DrzBS, then compared the inhibition effect of our CSO-SA/DrzBS complex to a common transfection reagent, Lipofectamine ${ }^{T M}$ 2000/DrzBS, on hepatitis B surface antigen expression. The synthesized CSO-SA assembles into micelles in an aqueous solution and exhibits excellent cytoplasmic targeting, and could protect DrzBS from degradation by ribonuclease. CSO-SA/DrzBS showed a higher inhibition rate (IR) than Lipofectamine ${ }^{T M}$ 2000/DrzBS. Moreover, at the same DrzBS concentration $\left(1.2 \mu \mathrm{mol} \mathrm{L}^{-1}\right)$, the maximum IR of CSO-SA/DrzBS micelles was 2.4-fold that of the Lipofectamine ${ }^{T M}$ 2000/DrzBS complex, and held on for 96 hours. Compared with Lipofectamine ${ }^{\text {TM }}$ 2000/DrzBS, CSO-SA/DrzBS achieved a higher HBV inhibition effect. This study demonstrates that CSO-SA micelles can serve as a potential vector for DrzBS and that CSO-SA/DrzBS micelles are a promising application for anti-HBV gene therapy.

\section{Introduction}

Hepatitis B, resulting from the infection of the hepatitis B virus (HBV), is one of the world's most common and serious infectious diseases. About two billion people globally have been exposed to the infection ${ }^{1}$ and more than 250 million people are suffering from chronic HBV infection. ${ }^{2-4}$ Twenty-five percent of all chronic HBV carriers develop serious liver diseases, such as chronic hepatitis, cirrhosis and primary hepatocellular carcinoma., HBV-related liver diseases still cause an estimated 500000 to one million deaths per year worldwide. ${ }^{7-9}$ Viral mutations ${ }^{\mathbf{1 0}}$ and patient immune tolerances to $\mathrm{HBV}$ antigens are the main causes of persistent chronic HBV infection. Therefore, reactivation of $\mathrm{HBV}$-specific immune responses is essential to reduce morbidity and mortality from chronic HBV infection.

$\mathrm{HBV}$ is an enveloped virus and can only infect humans and primates. The $\mathrm{S}$ gene codes for the viral envelope proteins

\footnotetext{
${ }^{a}$ Department of Pharmacy, the First Affiliated Hospital, College of Medicine, Zhejiang University, 79 Qingchun Road, Hangzhou 310003, P. R. China.E-mail: miaojing@zju. edu.cn; Fax: +86-571-87236675; Tel: +86-571-87236675

${ }^{b}$ Zhejiang Center of Laboratory Animals, Zhejiang Academy of Medical Sciences, 182 Tianmushan Road, Hangzhou 310007, P. R. China

${ }^{c}$ College of Pharmaceutical Sciences, Zhejiang University, 866 Yuhangtang Road, Hangzhou 310058, P. R. China
}

known as the hepatitis B surface antigen (HBsAg). Recent studies have shown that HBsAg accumulation was associated with specific viral mutations related to disease activities. ${ }^{11}$ In addition, when sufficient HBV envelope molecules are present, HBsAg is synthetized much more in excess than required for viral enveloping purposes, and most likely overwhelms any benefit of anti-HBs responses, causing immune tolerance. Persistence of HBsAg production is associated with an increased risk of liver cancer, even in the absence of productive HBV replication. ${ }^{12}$ Inhibition of HBsAg expression is a critical step in the functional control of HBV infection. It will unveil the unmaking of the existing anti-HBs response, which would favor clearance of virions, both infectious and noninfectious. ${ }^{13,14}$ In other words, the ability to clear HBsAg is the next frontier in hepatitis B therapeutics. The achievement of a 'functional cure' for chronic HBV infection with sustained HBsAg clearance, represents the next step in the pace towards HBV elimination. ${ }^{15}$

The discovery of DNAzymes breaks the traditional concept of protease-based biocatalysts. A DNAzyme is a structured DNA sequence with catalytic RNA-cleaving activity. The 10-23 DNAzyme consists of a catalytic domain formed by 15 deoxynucleotides in the center with recognition arms formed by seven to ten nucleotides on both sides. ${ }^{16}$ The enzyme specifically combines with the target mRNA in a highly sequencespecific manner through its recognition arms, while the 
central catalytic domain cleaves the mRNA target at purinepyrimidine junctions (A-U sites), thereby blocking the expression of the corresponding mRNA and inhibiting the target virus gene expression. ${ }^{\mathbf{1 7 - 2 1}}$

HBV is an attractive target for the 10-23 DNAzyme, and so we designed the HBV-specific 10-23 DNAzyme named DrzBS. Our previous research showed that DrzBS inhibited the expression of HBV $S$ genes by suppressing the corresponding antigen proteins, thus suggesting that DrzBS could be a powerful, specific, and effective form of anti-HBV gene therapy. In addition, DrzBS has a flexible design, independent of cellular mechanisms and does not require expensive chemical modifications when be used in vivo. And it does not affect the RNA of the host cell. Thus, DrzBS has a potential application value in anti-HBV gene therapy research. However, a major challenge for the application of the 10-23 DNAzyme is that it cannot replicate endogenously and, consequently, relies on exogenous delivery. Delivery of the 10-23 DNAzyme is limited by poor cellular uptake, rapid degradation by nucleases, and poor endosomal release. Therefore, development of a safe and efficient gene carrier is a prerequisite for successful gene therapy. ${ }^{22,23}$

Several non-viral vectors have been developed for DNA delivery that use a wide range of materials such as cationic liposomes, ${ }^{24,25}$ and natural or synthetic polymers. ${ }^{\mathbf{2 6 - 2 8}}$ Among these, chitosan has been extensively studied because it is inherently biocompatible, biodegradable, and has low cytotoxicity in comparison to other cationic polymers. ${ }^{23,29-31}$ Moreover, the positive surface charge and presence of easily modifiable primary amines and hydroxyl groups on the chitosan backbone make it an attractive tool for DNAzyme delivery. However, without laborious chemical modifications to its structure, it is of limited use as a gene delivery vehicle due to its low ability to efficiently transfect under physiological conditions. ${ }^{32,33}$ To address this problem, our recent studies ${ }^{34-36}$ have demonstrated that chitosan-g-stearic acid (CSOSA), which is synthesized via a reaction between the carboxyl group of stearic acid and the amine group of chitosan oligosaccharide in the presence of 1-(3-Dimethylamino-propyl)-3-ethylcarbodiimide (EDC), can greatly improve the cellular uptake and in vitro gene transfection efficacy of chitosan. ${ }^{37,38}$ This means that CSO-SA micelles could be an efficient approach for intracellular delivery of the 10-23 DNAzyme.

In this research, we designed and synthesized a kind of 10-23 DNAzyme, DrzBS, specific to the HBV $S$ gene open reading frame (ORF) $\mathrm{A}^{157} \mathrm{UG}$. The CSO-SA was achieved and bound with DrzBS by electrostatic interaction to form CSO-SA/DrzBS micelles, which could be an efficient approach for intracellular delivery of DrzBS. The characteristics and stability of CSOSA and CSO-SA/DrzBS micelles were studied, as well as the inhibition rate (IR) on HBsAg expression. Thus, we investigated CSO-SA as a gene vector and its therapeutic effect with DrzBS in HepG2.2.15 cells.

\section{Results and discussion}

\section{Synthesis and characterization of CSO-SA micelles}

In this study, the schematic diagram of CSO-SA/DrzBS targeting of cell uptake and intracellular release were shown in Fig. 1.
The synthesis route of the CSO-SA was shown in Fig. 2a, which was obtained by a coupling reaction between the amino groups of CSO and the carboxyl groups of SA in the presence of EDC. The final product, CSO-SA, was a white soluble powder after lyophilization. The chemical structure of CSO-SA was confirmed by ${ }^{1} \mathrm{H}$ NMR (Fig. 2b). The peaks at about $1.2 \mathrm{ppm}$ and $0.8 \mathrm{ppm}$ were attributed to the $-\mathrm{CH}_{2}$ and $-\mathrm{CH}_{3}$ groups respectively. These results proved the successful synthesis of the CSOSA micelles. The synthesized CSO-SA could be self-assembly in aqueous solution.

The average size and zeta potential of CSO-SA were $67.3 \pm$ $3.4 \mathrm{~nm}$ and $48.3 \pm 2.9 \mathrm{mV}$ respectively (Table 1 ). However, the values of PI were slightly large, which might due to the micelles dispersed in the deionized water where the $\mathrm{pH}$ and ionic strength were unstable. The spherical-like shape of the micelles were revealed with TEM images (Fig. 3). The CMC of CSO-SA was $75 \mu \mathrm{g} \mathrm{mL} \mathrm{L}^{-1}$.

\section{Characterization of CSO-SA/DrzBS micelles}

Based on the 10-23 DNAzyme molecular structure reported by Santoro and Joyce, ${ }^{\mathbf{1 6}}$ we designed and synthesized our 10-23 DNAzyme for the HBV S gene, named DrzBS (Fig. 2c). DrzBS is a DNA structure that can be combined with CSO-SA by electrostatic interaction to form CSO-SA/DrzBS micelles. The particle size and zeta potential of CSO-SA/DrzBS micelles are presented in Table 1. The average size was $158.0 \pm 2.5 \mathrm{~nm}$ and the zeta potential was $39.1 \pm 3.3 \mathrm{mV}$ in deionized water. The value of the zeta potential in the CSO-SA and the CSO-SA/DrzBS did not change much, which shows that the composition of the complex is hardly affected by the surface charge of the particles. The TEM photos (Fig. 3) indicate that CSO-SA/DrzBS micelles had a well-formed shape and compact structure.

\section{Tightlock experiment and DNase I protection assay}

Protection of DrzBS from nucleases as well as its polymer binding capability are both crucial factors for its efficient delivery to inhibit HBV gene expression. As the complexes pass through the cytoplasm, this protection effect is important. ${ }^{\mathbf{3 9 , 4 0}}$ This is because the enzymes and nucleic acids within the cytoplasm make the DrzBS complexes susceptible to hydrolytic and enzymatic degradation. CSO-SA exert the proper protection relied on appropriate N/P ratio. In our previous studies, ${ }^{\mathbf{4 1}}$ we have confirmed that CSO-SA micelles became to compact with 10-23 DNAzyme when N/P > 1.2, and further presented a good loading potency at the optimal N/P ratio of 10 . In addition, the migration of DrzBS within the CSO-SA/DrzBS complexes was entirely retarded in the loading slot when the N/P ratio was 10 . The DNase I protection assay also shown the same results. The naked DrzBS was significantly degraded, whereas DrzBS combined with the CSO-SA micelles were protected from the attack of DNase I at the N/P ratio of 10 as shown in Fig. 4.

\section{Cellular uptake}

The SD\% of CSO-SA, when concentrated in the nucleus and cytoplasm, were measured as $25.31 \%$ and $37.92 \%$ respectively. 


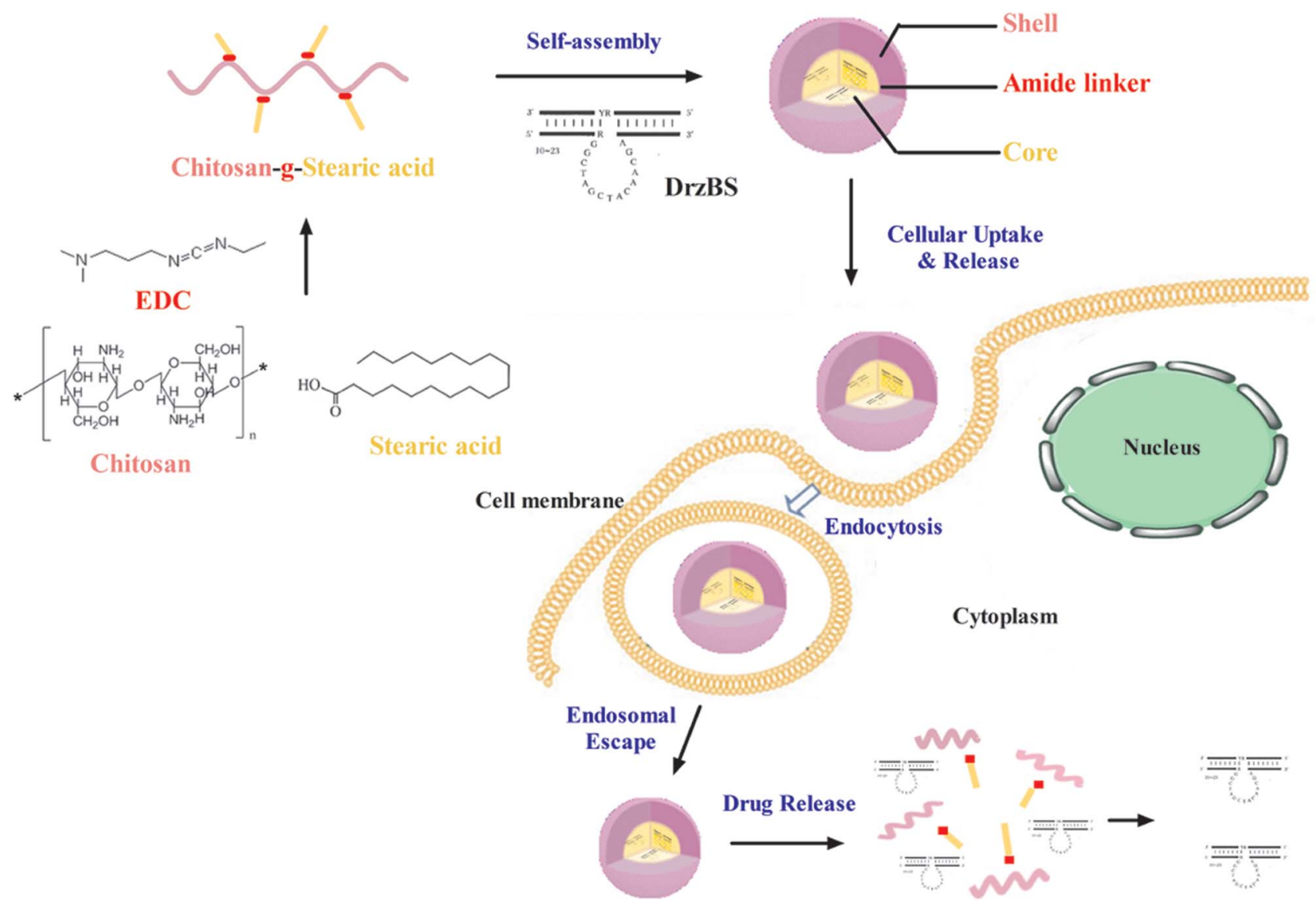

Fig. 1 The schematic diagram of passive targeting of cell uptake and intracellular release.

Since the inhibitory effect of DrzBS needs to be exerted in the cytoplasm, we chose the CSO-SA with an SD\% of $37.92 \pm 0.62 \%$.

In vitro cellular uptake of FITC-labeled micelles on HepG2.2.15 cells were visualized by fluorescence microscope at $2 \mathrm{~h}$ and $4 \mathrm{~h}$. Fig. 5a shows that the cellular uptake of FITClabeled CSO-SA micelles in cell lines was time dependent. The polymer showed favorable cellular internalization, which mainly depended on the hydrophobic core composed of SA and moderate surface charge. The CLSM results were also shown that the CSO-SA with an $\mathrm{SD} \%$ of $37.92 \pm 0.62 \%$ has the ability to target cytoplasm, and the cellular uptake of the CSO-SA micelles is time dependent (Fig. 5b). These results suggested that CSO-SA micelles were efficient intracellular delivery systems and could improve the cellular uptake into HepG2.2.15 cells.

\section{In vitro cytotoxicity}

A good quality vector should be biocompatible and not damage the cell structure or its normal function. To examine the cytotoxicity of the CSO-SA and Lipofectamine ${ }^{\mathrm{TM}} 2000$, cell viability was determined by MTT assay. Fig. 6a shows that CSO-SA micelles exhibit excellent cell compatibility in HepG2.2.15 and L02 (non-tumour hepatocytes) cells, whereas the cytotoxicity of Lipofectamine ${ }^{\mathrm{TM}} 2000$ was found to be much higher, as previously reported. ${ }^{42}$ The half maximal inhibitory concentration $\left(\mathrm{IC}_{50}\right)$ value in HepG2.2.15 cells were $6.8 \mu \mathrm{g} \mathrm{mL} L^{-1}$ for

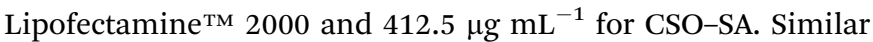
to that, the $\mathrm{IC}_{50}$ value in $\mathrm{L} 02$ cells were $4.2 \mu \mathrm{g} \mathrm{mL} \mathrm{L}^{-1}$ and $254.0 \mu \mathrm{g}$ $\mathrm{mL}^{-1}$, respectively. Its safety provided more extensive dosage range for DNAzymes transfection. The survival rates of the CSOSA/DrzBS micelles were all approximately $100 \%$ in L02 cells and above $75 \%$ in HepG2.2.15 cells at various concentrations of DrzBS (DrzBS concentration $=0.1,0.6,1.2,1.8,2.4,3.6,4.8,9.6$ $\mu \mathrm{mol} \mathrm{L}^{-1}$ ). As for Lipofectamine ${ }^{\mathrm{TM}} 2000$, the cell viability drastically decreased (Fig. 6b).

The cytotoxicity of Lipofectamine ${ }^{\mathrm{TM}} 2000$ may be a result of its high positive surface charges and its aggregation on cell surfaces, thus impairing important membrane functions. ${ }^{28,43,44}$ In contrast, the sources for CSO-SA synthesis, CSO and SA, were either biodegradable, low toxic or biocompatible. Compared with Lipofectamine ${ }^{\mathrm{TM}} 2000$, CSO-SA micelles had relatively low cytotoxicity. Together, these internalization and cytotoxicity results verified that the CSO-SA is safe and has potential as an anti-HBV gene therapy.

\section{Transfection and inhibitory effects of DrzBS on HBsAg expression in HepG2.2.15 cells}

Our experiments showed that the inhibition percentages of HBsAg expression in the HepG2.2.15 cells by our CSO-SA complexes were $16.7 \pm 3.2 \%$ (for the $\mathrm{N} / \mathrm{P}$ ratio $=5: 1$ ) and 51.3 $\pm 4.6 \%$ (for the N/P ratio $=10: 1)(p<0.001$, Fig. $7 \mathrm{a})$. This confirms that the $\mathrm{N} / \mathrm{P}$ ratio of $10: 1$ achieves the best 
a

Step 1<smiles>CCNCCCNCCCN(C)C</smiles>

EDC

но

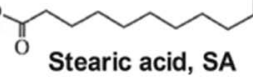

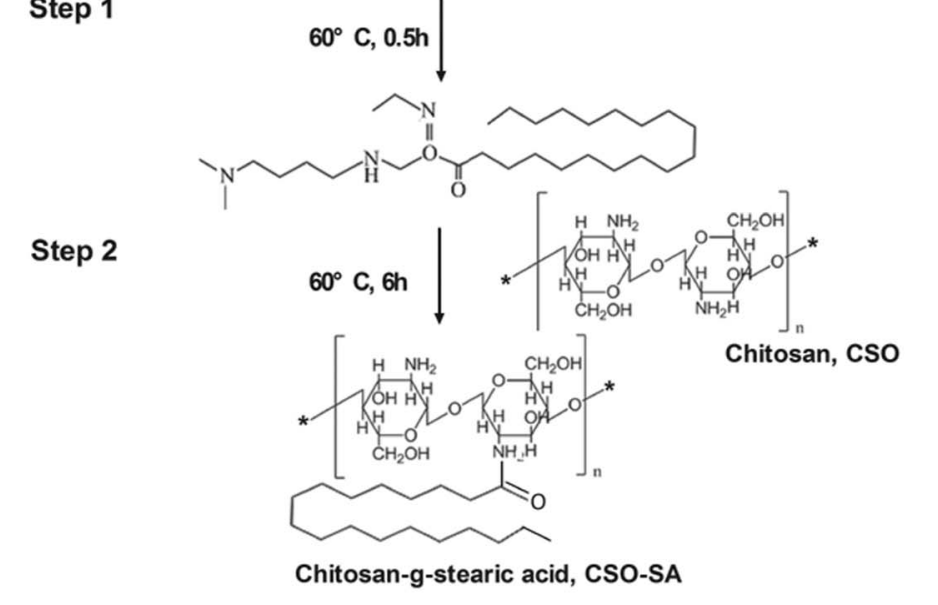

C

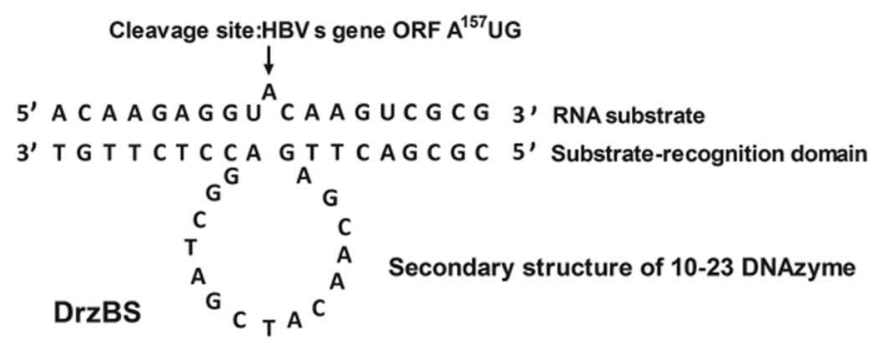

b

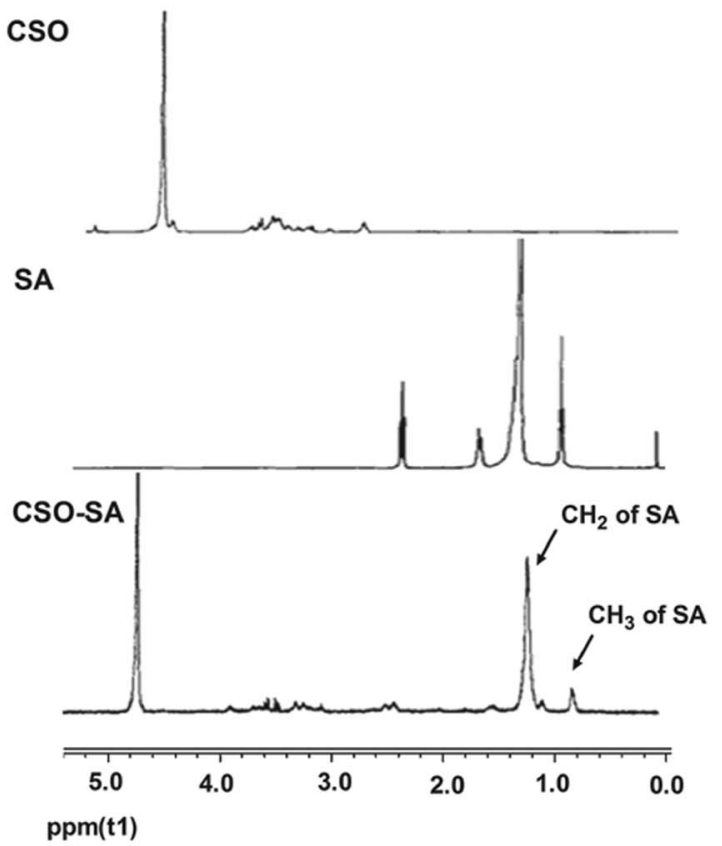

Fig. 2 The schematic diagram of chitosan-g-stearic acid (CSO-SA). (a) Sequence of DrzBS and RNA substrates (arrow indicating cleavage site). (b) Synthesis route of CSO-SA. (c) ${ }^{1} \mathrm{H} N M R$ spectra, from top to bottom: chitosan, stearic acid, and CSO-SA. The signals assigned to the $-\mathrm{CH}_{2}{ }^{-}$ (1.2 ppm) and $-\mathrm{CH}_{3}-(0.8 \mathrm{ppm}) \mathrm{SA}$ were detectable on the CSO-SA spectra.

Table 1 Characteristics of CSO-SA and CSO-SA/DrzBS micelles ( $n=$ 3)

\begin{tabular}{lcll}
\hline Polymers & $\begin{array}{l}\text { Volume average } \\
\text { diameter }(\mathrm{nm})\end{array}$ & Zeta potential $(\mathrm{mV})$ & PI \\
\hline CS-SA & $67.3 \pm 3.4$ & $48.3 \pm 2.9$ & $0.57 \pm 0.06$ \\
CS-SA/DrzBS & $158.0 \pm 2.5$ & $39.1 \pm 3.3$ &
\end{tabular}

intracellular efficacy. It is also worth noticing that the inhibition percentage in the CSO-SA group was higher than that in Lipofectamine $^{\mathrm{TM}} 2000(35.8 \pm 2.5 \%)$ at the similar N/P ratio of $10: 1(51.3 \pm 4.6 \%, p<0.01)$. These results indicate that, at the $\mathrm{N} / \mathrm{P}$ ratio of $10: 1, \mathrm{CSO}-\mathrm{SA} / \mathrm{DrzBS}$ micelles can efficiently transfect into cells and release DrzBS into the cytoplasm to effectively inhibit HBsAg levels.

On the basis of the above experiment, different concentrations of DrzBS were selected to evaluate the IR on HBsAg for the same incubation time of $48 \mathrm{~h}$. Within a concentration range of 0.2 to $1.8 \mu \mathrm{mol} \mathrm{L} \mathrm{L}^{-1}$, the IR on HBsAg expression of DrzBS delivered by Lipofectamine ${ }^{\mathrm{TM}} 2000$ increased from $17.3 \pm 3.8 \%$ to $44.1 \pm 3.4 \%$, while the IR on HBsAg expression of DrzBS delivered by CSO-SA micelles increased from $9.2 \pm 3.6 \%$ to 73.5 $\pm 3.0 \%$, with both complexes showing a distinct dosedependent effectiveness (Fig. 7b). At low concentrations (0.2 $\left.\mu \mathrm{mol} \mathrm{L}{ }^{-1}\right)$, the IR of CSO-SA/DrzBS $(9.2 \pm 3.6 \%)$ was lower than that of Lipofectamine ${ }^{\mathrm{TM}} 2000 /$ DrzBS $(17.3 \pm 3.8 \%)$, probably because the release of the drug from the CSO-SA micelles did not reach its intracellular peak, whereas the Lipofectamine ${ }^{\mathrm{TM}}$ 2000 complex had reached its peak. But, when the concentration of DrzBS reached $0.6 \mu \mathrm{mol} \mathrm{L}{ }^{-1}$, the DrzBS delivered by the CSO-SA micelles exhibited much higher efficiency for inhibiting HBsAg expression than that delivered by Lipofectamine ${ }^{\mathrm{TM}}$ $2000(33.3 \pm 8.9 \%$ vs. $29.7 \pm 9.4 \%)$. And when the concentration of DrzBS reached $1.2 \mu \mathrm{mol} \mathrm{L}{ }^{-1}$, the IR of the CSO-SA/DrzBS micelles with the value of $69.6 \pm 3.2 \%$ which was significantly higher than that of Lipofectamine ${ }^{\mathrm{TM}}$ 2000/DrzBS complexes $(36.9 \pm 3.9 \%, p<0.001)$. Remarkably, at further increases of DrzBS dose concentrations, IR growth became more slowly, and platform-like effectiveness was observed. These results confirmed the capacity of the CSO-SA/DrzBS micelles selective release DrzBS in the cytoplasm.

To further understand the potential mechanism of the decreased cytotoxicity and intracellular effects observed with CSO-SA/DrzBS micelles, we examined their inhibitory effect on $\mathrm{S}$ gene expression over time and at the same concentration $(1.2$ $\mu \mathrm{mol} \mathrm{L} \mathrm{L}^{-1}$ ). Fig. 7c showed the HBsAg inhibition effect after transfection. It was obvious that DrzBS cannot inhibit HBsAg itself, and the CSO-SA has a slight inhibition of HBsAg but 
CSO-SA

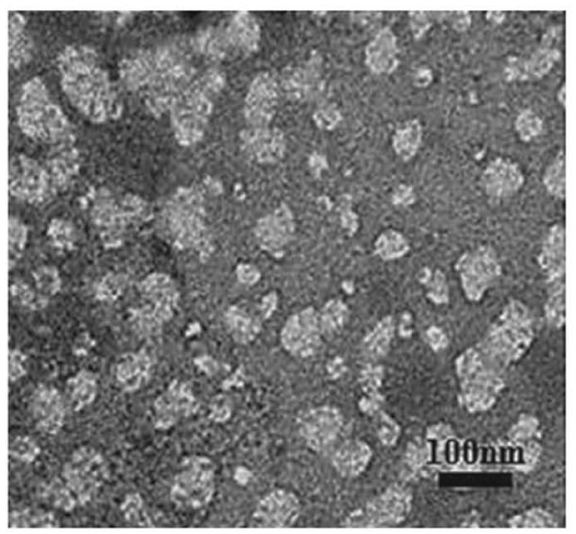

CSO-SA/DrzBS

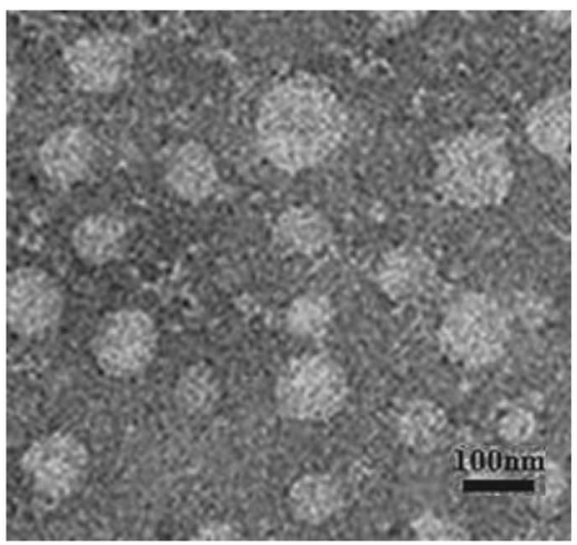

Fig. 3 TEM images of the CSO-SA and CSO-SA/DrzBS micelles. Scale bar, $100 \mathrm{~nm}$.

much lower than the CSO-SA/DrzBS micelles. CSO-SA/DrzBS micelles displayed a significant HBsAg inhibition compared with that of Lipofectamine ${ }^{\mathrm{TM}}$ 2000/DrzBS complexes. The IR of Lipofectamine $^{\mathrm{TM}}$ 2000/DrzBS on HBsAg expression increased during the first $48 \mathrm{~h}$ and reached a maximum IR of $34.8 \pm 0.6 \%$ at $48 \mathrm{~h}$, due to the relatively high cell transduction efficiency of Lipofectamine 2000 in HepG2.2.15 cells and the peak expression of DrzBS in the cells. However, after $48 \mathrm{~h}$, the IR began to descend, and at $60 \mathrm{~h}$, the IR started to rapidly decrease, possibly due to the system toxicity of Lipofectamine ${ }^{\mathrm{TM}} 2000$. On the other hand, the IR of HBsAg expression treated by CSO-SA/ DrzBS micelles continued to increase with the incubation time for $72 \mathrm{~h}(84.1 \pm 2.6 \%)$ and remained stable. When viewed with CSO-SA/DrzBS micelles for $96 \mathrm{~h}$, the IR value was $83.5 \pm 2.7 \%$, which was significantly higher than that of Lipofectamine ${ }^{\mathrm{TM}}$ 2000/DrzBS $(18.6 \pm 2.4 \%, p<0.001)$. These results demonstrate that CSO-SA possesses a higher efficiency and a lower cytotoxicity for inhibiting HBsAg expression than Lipofectamine ${ }^{\mathrm{TM}}$ 2000. Furthermore, the drug release in intracellular compartments made the free DrzBS produce a better pharmacological action.

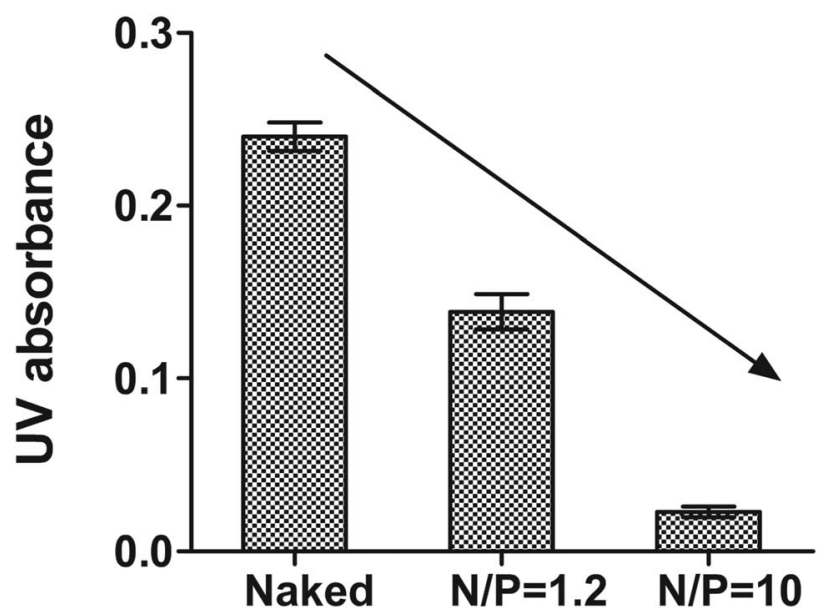

Fig. 4 UV absorbance at $260 \mathrm{~nm}$ in DNase I protection assay with or without CSO-SA micelles (reaction time was $15 \mathrm{~min}$, mean $\pm \mathrm{SD}, n=3$ ).

\section{Experimental}

\section{Materials}

Chitosan oligosaccharide (CSO) of low molecular weight ( $\mathrm{MW}=$ $18.4 \mathrm{kDa}$ ) was obtained by enzymatic degradation of chitosan (95\% deacetylated, MW $=450 \mathrm{kDa}$ ), and supplied by Yuhuan Marine Biochemistry Co., Ltd. (Zhejiang, China). Stearic acid (SA) was purchased from Shanghai Chemical Reagent Co., Ltd. (Shanghai, China). EDC hydrochloride and pyrene were provided by Aladdin Reagent Co., Ltd. (Shanghai, China). Methylthiazol-tetrazolium (MTT), 2,4,6-trinitrobenzenesulfonic acid (TNBS), and 5-fluorescein isothiocyanate (FITC) were provided by Sigma Chemical Co. Lipofectamine ${ }^{\mathrm{TM}} 2000$ and Opti-MEMI medium were supplied by Invitrogen Corporation (Carlsbad, CA, USA). Trypsin and Dulbecco's minimum essential medium (DMEM) were purchased from Gibco (Merelbeke, Belgium). HepG2.2.15 cells were provided by the State Key Laboratory for Diagnosis and Treatment of Infectious Diseases of Zhejiang Province (Hangzhou, China). The HBsAg quantitative radioimmunoassay kit was purchased from Weifang $3 \mathrm{~V}$ diagnostic technology company (Shandong, China).

\section{Design and synthesis of 10-23 DNAzyme}

Based on the molecular structure of 10-23 DNAzyme, we designed a 10-23 DNAzyme, named DrzBS, using Watson-Crick base pairing, and specific to the HBV S gene (Genbank gi:59429) ORF $\mathrm{A}^{157}$ UG. We consigned its synthesis to Sangon Biological Engineering Technology \& Service Co., Ltd. (Shanghai, China). DrzBS contains the 15-nucleotide catalytic sequence GGCTAGCTACAACGA and has nine oligonucleotides on either side. Named DrzBS-9, this DNAzyme can specifically recognize the HBV S gene ORF $A^{157}$ UG sequence from the 148th to 166th nucleotide.

\section{Synthesis of chitosan- $g$-stearic acid (CSO-SA)}

Chitosan oligosaccharide was prepared according to the previous study. ${ }^{45} \mathrm{CSO}-\mathrm{SA}$ was then synthesized via a reaction of the carboxyl group of stearic acid with the amine group of the chitosan oligosaccharide in the presence of EDC. ${ }^{46}$ Briefly, SA 
a
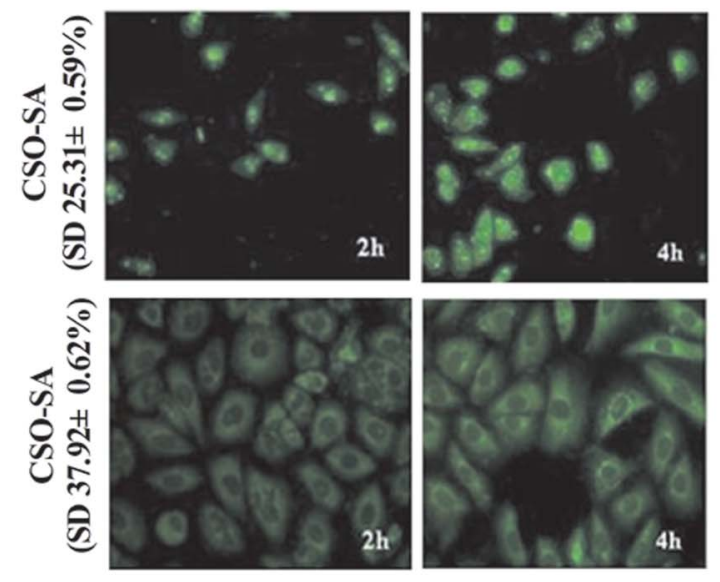

b
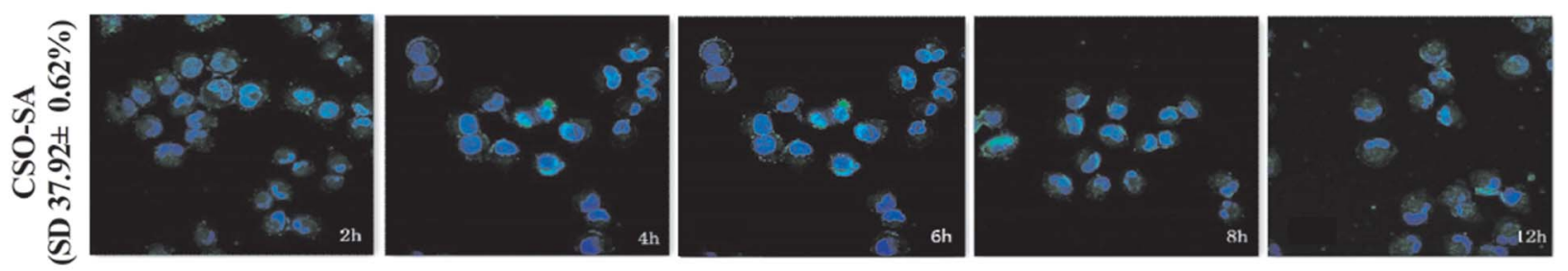

Fig. 5 Representative confocal fluorescent images of the cellular uptake of FITC-CSO-SA/DrzBS on HepG2.2.15 cells. The CSO-SA concentration was $100 \mu \mathrm{g} \mathrm{mL}^{-1}, \mathrm{SD} \%$ is the degrees of amino-substitution.

a

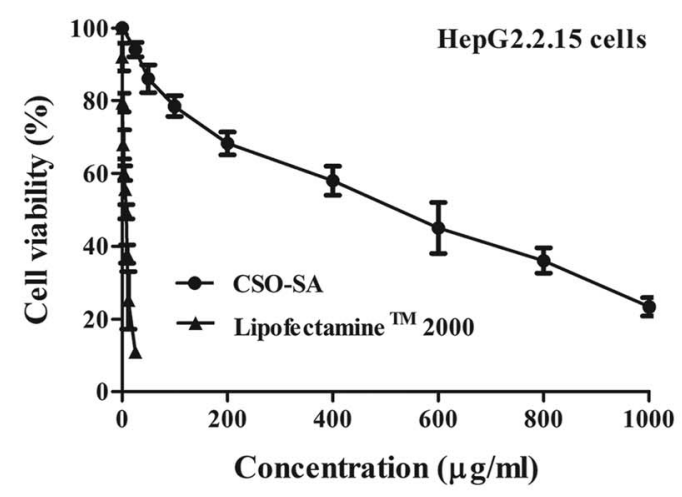

b

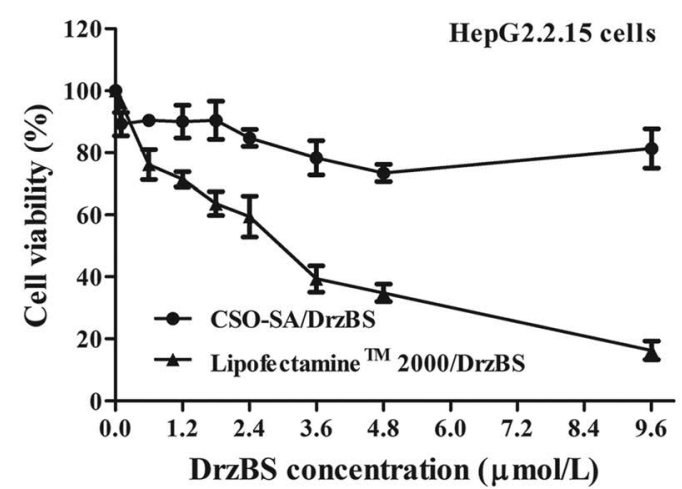

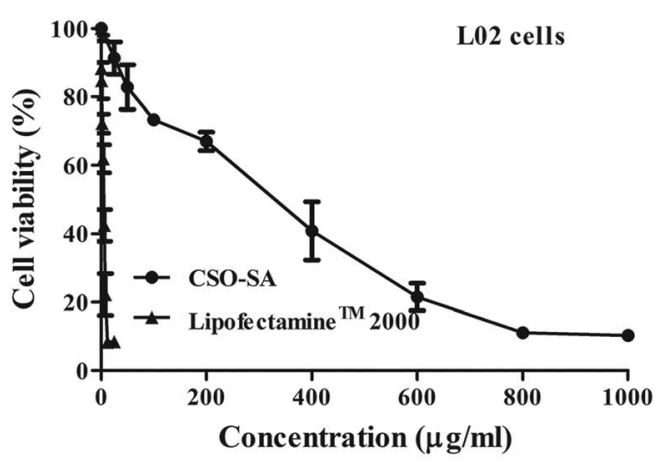

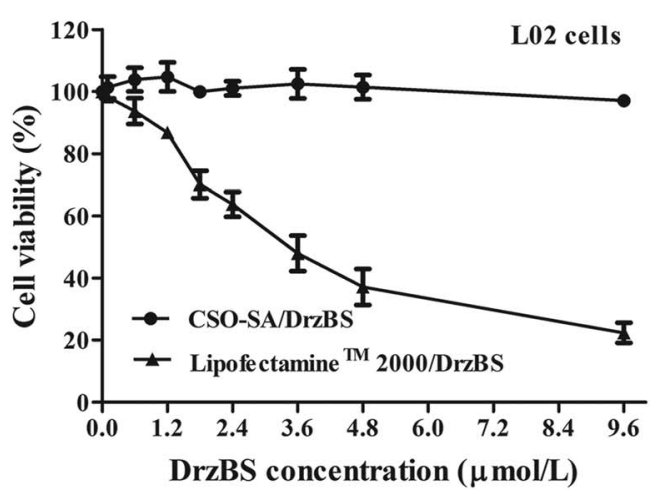

Fig. 6 (a) Cell viabilities of Lipofectamine ${ }^{T M} 2000$ and CSO-SA on HepG2.2.15 cells and L02 cells. (b) Cell viabilities of Lipofectamine ${ }^{\text {TM }} 2000 /$ DrzBS and CSO-SA/DrzBS on HepG2.2.15 cells and L02 cells with different DrzBS concentration. Error bars represent the standard deviation (mean $\pm \mathrm{SD}, n=3$ ). 
a

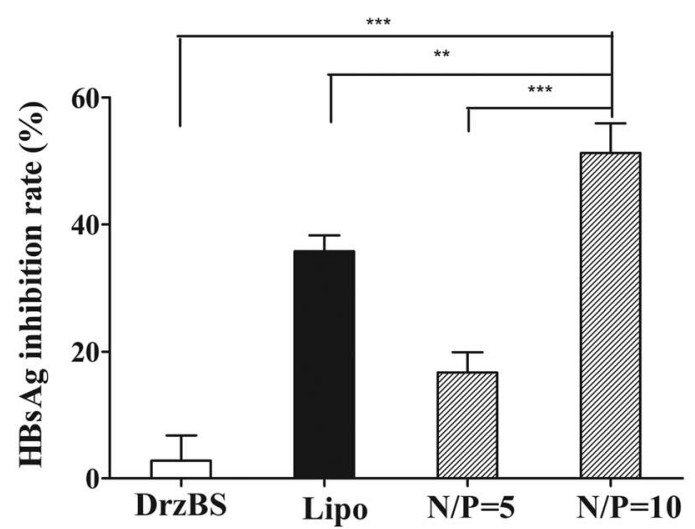

b

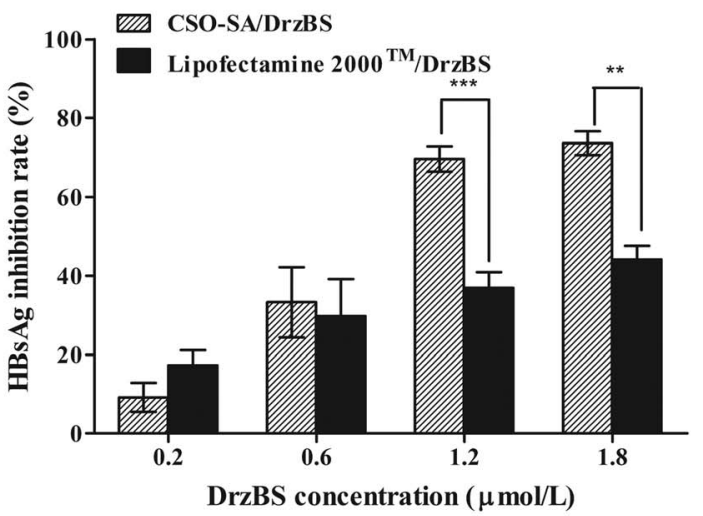

C

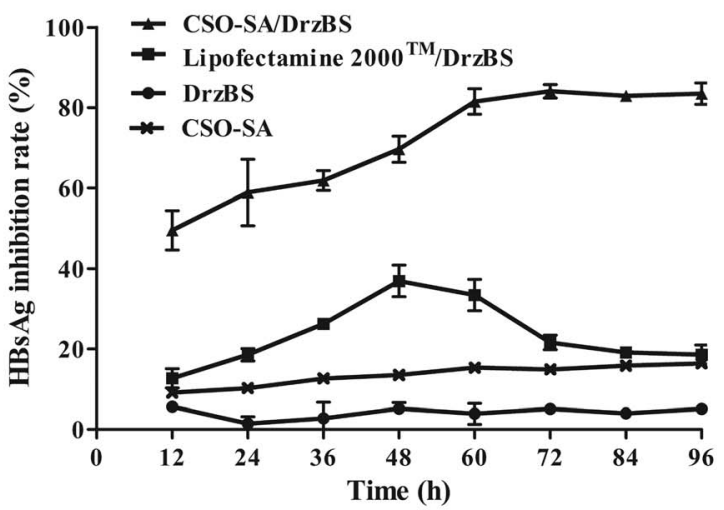

Fig. $7 \mathrm{HBsAg}$ inhibition effect of DrzBS transfected by the polymer complexes. (a) Inhibitory effect of CSO-SA/DrzBS complex on HepG2.2.15 cells for $48 \mathrm{~h}$ (mean $\pm \mathrm{SD}, n=3$ ). Lipo was the abbreviation for Lipofectamine ${ }^{T M}$ 2000. (b) For the same incubation time of $48 \mathrm{~h}$ with different DrzBS concentration (mean $\pm \mathrm{SD}, n=3, * * p<0.01, * * * p$ $<0.001$ ). (c) In the same DrzBS concentration of $1.2 \mu \mathrm{mol} \mathrm{L}{ }^{-1}$ for different incubation time. The error is the standard deviation from the mean (mean $\pm \mathrm{SD})$.

and EDC were dissolved in acetone and then added into an ethanol-acetone mixed solvent (ethanol : acetone ratio $=3: 7$, $\mathrm{v} / \mathrm{v}$ ). The mixture was stirred for $0.5 \mathrm{~h}$ at $60{ }^{\circ} \mathrm{C}$. CSO (MW 18.4 $\mathrm{kDa}$ ) was then dissolved in hot water, after which the SA/EDC mixture was added dropwise. The reaction solution was stirred for another $6 \mathrm{~h}$ and was then dialyzed against deionized water using a dialysis membrane (molecular weight cut-off
(MWCO): 7 kDa, Spectrum Laboratories, Laguna Hills, CA) for $48 \mathrm{~h}$. Finally, the product was collected by lyophilization followed by a further wash with ethanol to remove products. The washed product was then collected by lyophilization again.

\section{Preparation of Lipofectamine ${ }^{\mathrm{TM}}$ 2000/DrzBS and CSO-SA/ DrzBS micelles}

The Lipofectamine ${ }^{\mathrm{TM}}$ 2000/DrzBS complex was prepared by gently mixing DrzBS with Lipofectamine ${ }^{\mathrm{TM}} 2000$ in $50 \mu \mathrm{L}$ OPTIMEMI (total volume: $100 \mu \mathrm{L}$ ), followed by incubation for $20 \mathrm{~min}$ at room temperature.

CSO-SA solutions with different concentrations (dissolved in $25 \mathrm{mM}$ sodium acetate buffer, pH 5.5) were first purified with a $0.22 \mathrm{~m}$ Millipore filter. CSO-SA/DrzBS micelles with various N/ $\mathrm{P}$ ratios (The N/P ratio is the number of unreacted free primary amines of CSO-SA to the number of phosphate groups of DrzBS) were prepared by mixing appropriate volumes of CSO-SA micelle solutions with DrzBS solutions (diluted with water, 500 $\mu \mathrm{g} \mathrm{mL} \mathrm{L}^{-1}$ ). After mixing in a vortex for $30 \mathrm{~s}$, the mixtures were incubated for $30 \mathrm{~min}$ at $37^{\circ} \mathrm{C}$. Electrophoresis was then carried out with a current of $100 \mathrm{~V}$ for $20 \mathrm{~min}$ in a TAE buffer solution (40 mM Tris-HCl, 1\% (v/v) acetic acid, 1 mM EDTA).

\section{Characterization of CSO-SA and CSO-SA/DrzBS micelles}

The chemical structure of CSO-SA was determined with ${ }^{1} \mathrm{H}$ nuclear magnetic resonance (NMR) spectroscopy, and this chemical was dispersed in $\mathrm{D}_{2} \mathrm{O}$ at $\mathrm{pH} 7$ and $25^{\circ} \mathrm{C}$. The substitution degree ( $\mathrm{SD} \%$ ) of amino groups of $\mathrm{CSO}-\mathrm{SA}$, defined as the molar ratio of stearate to anhydroglucosidic units in chitosan oligosaccharide, was detected with TNBS, and the ultraviolet (UV) absorbance of the final reaction mixture at $344 \mathrm{~nm}$ was measured by UV spectroscopy (TU-1800PC, Beijing Purkinje General Instrument Co., Ltd., China). The critical micelle concentration (CMC) of CSO-SA was measured by pyrene fluorescence using a fluorometer (F-2500, Hitachi Co., Japan). The intensity ratio $\left(I_{1}: I_{3}\right)$ of the first peak $\left(I_{1}, 374 \mathrm{~nm}\right)$ to the third peak $\left(I_{3}, 385 \mathrm{~nm}\right)$ in the pyrene emission spectra was analyzed to calculate the CMC.

The size and zeta potential of CSO-SA micelles and CSO-SA/ DrzBS micelles were measured by dynamic light scattering (Zetasizer 3000HS, Malvern Instruments Ltd., UK) in deionized water. Their morphology was examined by transmission electronic microscopy (TEM, Stereoscan, Leica, England).

\section{DNase I protection assay}

The DNase I protection assay was performed to investigate the efficiency of the CSO-SA micelles protecting the DrzBS from enzymatic degradation by DNase I. The CSO-SA/DrzBS micelles with 1.2 and 10 of N/P ratios were incubated in a buffer solution (10 mM Tris-HCl, $150 \mathrm{mM} \mathrm{NaCl,} 1 \mathrm{mM} \mathrm{MgCl}_{2}, \mathrm{pH} 7.4$ ) with DNase I (25 units), then shaken at $100 \mathrm{rpm}$ for $30 \mathrm{~min}$ at $37^{\circ} \mathrm{C}$. Electrophoresis was conducted in $1 \%$ agarose gel with a voltage of $100 \mathrm{~V}$. UV absorbance was measured by UV spectrophotometer at $260 \mathrm{~nm}$ (UV-2450, Shimadzu, Japan). 


\section{Cell culture}

Human normal liver cells (L02 cell) were obtained from American Type Culture Collection (ATCC, Rockville, MD, USA). HBVtransfected human hepatoma cells (HepG2.2.15 cells) were obtained from the Cell Bank of the Chinese Academy of Sciences (Shanghai, China) and cultured in a biological safety protection third-level laboratory (P3 laboratory, The First Affiliated Hospital, Zhejiang University). All cells lines were cultured at $37^{\circ} \mathrm{C}$ in DMEM supplemented with $10 \%$ fetal bovine serum in a humidified atmosphere containing $5 \% \mathrm{CO}_{2}$.

\section{Cellular uptake}

To investigate the cell internalization, FITC-labeled CSO-SA was prepared. First, a FITC $\left(\mathrm{C}_{21} \mathrm{H}_{11} \mathrm{NO}_{5} \mathrm{~S}\right)$ ethanol solution was added dropwise into a $1.0 \mathrm{mg} \mathrm{mL} \mathrm{mL}^{-1} \mathrm{CSO}-\mathrm{SA}$ aqueous solution (CSO-SA : FITC ratio $=1: 1, \mathrm{~mol}: \mathrm{mol})$, then stirred overnight at $400 \mathrm{rpm}$ in an aphotic environment and subsequently dialyzed $(\mathrm{MWCO}=3.5 \mathrm{kDa}$ ) against pure water for $6 \mathrm{~h}$ to remove ethanol and unreacted FITC. The HepG2.2.15 cells were then incubated with these FITC-labeled CSO-SA micelles $(\mathrm{N} / \mathrm{P}$ ratio $=$ 10), at a CSO-SA concentration of $100 \mu \mathrm{g} \mathrm{mL} \mathrm{m}^{-1}$, in a growth medium for $2 \mathrm{~h}, 4 \mathrm{~h}$ and then washed three times with a phosphate buffered solution (PBS) (pH 7.4). We then used a fluorescence microscope (OLYMPUS America, Melville, NY) to observe the internalization ability of CSO-SA and the cellular uptake of the CSO-SA/DrzBS micelles.

\section{In vitro cytotoxicity assay}

In vitro cytotoxicity of Lipofectamine ${ }^{\mathrm{TM}} 2000$, CSO-SA and their complexes were evaluated by MTT assay on HepG2.2.15 cells and L02 cells. Briefly, $1.0 \times 10^{4}$ cells were plated in 96-well plates and incubated for $48 \mathrm{~h}$ to allow the cells to attach. Various concentrations of Lipofectamine ${ }^{\mathrm{TM}} 2000$, CSO-SA and the controls were added. After $48 \mathrm{~h}, 50 \mu \mathrm{g}$ MTT solution $(5 \mathrm{mg}$ $\mathrm{mL}^{-1}$ ) was added to each well, and the cells were incubated for a further $4 \mathrm{~h}$. After the unreduced MTT and medium were removed, each well was washed with $100 \mu \mathrm{L}$ of PBS, and then $200 \mu \mathrm{L}$ of dimethyl sulfoxide (DMSO) was added to dissolve the formazan. Finally, the absorbance of each well was detected with a microplate reader (BioRad, Model 680, USA) at $570 \mathrm{~nm}$. The survival percentage was calculated as compared to mocktreated cells (100\% survival). The reported data represent the means of the triplicate measurements and the standard errors of the means were less than $15 \%$. The transfection cytotoxicity of Lipofectamine $^{\mathrm{TM}}$ 2000/DrzBS and CSO-SA/DrzBS (N/P = 10) complexes containing various DrzBS concentration were also measured as above.

\section{Transfection and inhibitory effects of DrzBS on HBsAg expression in HepG2.2.15 cells}

HepG2.2.15 cells were seeded in 24-well plates at a density of 5 $\times 10^{4}$ cells per well. After transfection with CSO-SA/DrzBS micelles at N/P ratios of 5 and 10 for $48 \mathrm{~h}$, HepG2.2.15 cell culture supernatant was harvested. Naked DrzBS was used as the negative control. The Lipofectamine ${ }^{\mathrm{TM}}$ 2000/DrzBS complex served as the positive control. The level of HBsAg in the cell culture supernatant was determined by radioimmunoassay (under the same DrzBS concentration of $1.0 \mu \mathrm{mol} \mathrm{L}^{-1}$ ) to quantify the cell transfection and intracellular efficacy.

To evaluate the therapeutic efficiency of the CSO-SA/DrzBS delivery system, HepG2.2.15 cells were seeded at $1.0 \times 10^{5}$ cells per well in a 24-well plate and grown for $48 \mathrm{~h}$ to allow the cells to attach. After the medium was replaced with $0.5 \mathrm{~mL}$ OPTI-MEMI, different amounts of CSO-SA/DrzBS micelles (N/P ratio $=10$ ) and Lipofectamine ${ }^{\mathrm{TM}}$ 2000/DrzBS (the positive control) were added to each well and incubated with the cells for $6 \mathrm{~h}$. The medium was then replaced with $1 \mathrm{~mL}$ of fresh complete medium, and the incubation was continued for a further 12 to $96 \mathrm{~h}$ at $37^{\circ} \mathrm{C}$. All the experiments were performed in triplicate. The culture media from the wells were collected for virological assessment. HBsAg production was determined by commercial enzyme immunoassay kits (AXSYM System, Abbott, Wiesbaden, Germany).

\section{Conclusions}

In this study, the CSO-SA was synthesized and evaluated as a novel non-viral DNA carrier by binding with a kind of 10-23 DNAzyme named DrzBS, which specifically targeted the expression of HBV S genes. The CSO-SA micelles demonstrated superior ability to form nanoscale complexes with DrzBS and had suitable physicochemical properties for DNAzyme delivery, thus finally leading to enhanced gene transfer efficiency, protected DrzBS from degradation, and effectively released DrzBS after cellular uptake into HepG2.2.15 cells. The CSO-SA/DrzBS micelles significantly inhibited HBsAg expression in HepG2.2.15 cells compared to Lipofectamine ${ }^{\mathrm{TM}}$ 2000/DrzBS micelles $(p<0.001)$. Most importantly, the inhibitory effect of the CSO-SA/DrzBS micelles on HBV S gene expression could be prolonged to $96 \mathrm{~h}$, which is crucial for anti-HBV gene therapy. In summary, DrzBS delivered by the CSO-SA showed obvious inhibitory effects of HBsAg expression and therapeutic effects, indicating that the CSO-SA was a potential vector for gene therapy and might be used for the successful treatment of chronic HBV carriers.

\section{Conflicts of interest}

There are no conflicts to declare.

\section{Acknowledgements}

This work was supported by the Zhejiang Provincial Natural Science Foundation of China (Qing Shan $\mathrm{Hu}$ Science\&Technology City Union Foundation, LQY19H300001), the National Nature Science Foundation of China (81402862 and 81503256), the Hospital Pharmacy Special Research Project of Zhejiang Pharmaceutical Association (2018ZYY02, 2016ZYY01), and the Medical and Health Science and Technology Project of Zhejiang Province (No. 2018247580). 


\section{References}

1 World Health Organization, Guidelines for the Prevention, Care and Treatment of Persons with Chronic Hepatitis $B$ Infection, Geneva, 2015.

2 R. Orlando, M. Foggia, A. E. Maraolo, S. Mascolo, G. Palmiero, O. Tambaro and G. Tosone, Eur. J. Clin. Microbiol. Infect. Dis., 2015, 34, 1059-1070.

3 S. Mittal and H. B. El-Serag, J. Clin. Gastroenterol., 2013, 47(suppl.), S2-S6.

4 P. Karayiannis, Hepatol. Int., 2017, 11, 500-508.

5 Y. F. Liaw, M. R. Brunetto and S. Hadziyannis, Antiviral Ther., 2010, 15, 25-33.

6 The Korean Association for the Study of the Liver (KASL), KASL clinical practice guidelines: management of chronic hepatitis B, Clin. Mol. Hepatol., 2016, 22, 18-75.

7 F. Cui, J. Drobeniuc, S. C. Hadler, Y. J. Hutin, F. Ma, S. Wiersma, F. Wang, J. Wu, H. Zheng, L. Zhou and S. Zuo, Vaccine, 2013, 31(Suppl 9), J79-J84.

8 W. Cao, Z. Qiu, T. Zhu, Y. Li, Y. Han and T. Li, J. Clin. Virol., 2014, 61, 40-46.

9 E. F. Duffell and M. J. van de Laar, Euro Surveill., 2015, 20, 17-24.

10 Y. H. Hsieh, I. J. Su, H. C. Wang, W. W. Chang, H. Y. Lei, M. D. Lai, W. T. Chang and W. Y. Huang, Carcinogenesis, 2004, 25, 2023-2032.

11 Y. Li, Q. Cai, Q. Xie, Y. Zhang, X. Meng and Z. Zhang, Med. Sci. Monit., 2017, 23, 1385-1393.

12 T. C. Tseng, C. J. Liu, H. C. Yang, T. H. Su, C. C. Wang, C. L. Chen, S. F. Kuo, C. H. Liu, P. J. Chen, D. S. Chen and J. H. Kao, Gastroenterology, 2012, 142, 1140-1149.

13 S. Fourati and J. M. Pawlotsky, F1000Research, 2016, 5, 2243.

14 H. Ward, L. Tang, B. Poonia and S. Kottilil, Future Microbiol., 2016, 11, 1581-1597.

15 V. Soriano, P. Barreiro, L. Benitez, J. M. Pena and C. de Mendoza, Expert Opin. Invest. Drugs, 2017, 26, 843-851.

16 S. W. Santoro and G. F. Joyce, Proc. Natl. Acad. Sci. U. S. A., 1997, 94, 4262-4266.

17 N. Singh, A. Ranjan, S. Sur, R. Chandra and V. Tandon, J. Biosci., 2012, 37, 493-502.

18 D. Kumar, I. Chaudhury, P. Kar and R. H. Das, J. Gastroenterol. Hepatol., 2009, 24, 872-878.

19 V. Sood, N. Gupta, A. S. Bano and A. C. Banerjea, Oligonucleotides, 2007, 17, 113-121.

20 H. Unwalla, S. Chakraborti, V. Sood, N. Gupta and A. C. Banerjea, Antiviral Res., 2006, 72, 134-144.

21 W. Hou, J. E. Wo, K. Z. Liu, M. W. Li, L. W. Chen, Z. R. Hu, R. H. Liu and M. J. Hu, Zhonghua Neike Zazhi, 2006, 45, 396399.

22 Y. K. Kim, A. Minai-Tehrani, J. H. Lee, C. S. Cho, M. H. Cho and H. L. Jiang, Int. J. Nanomed., 2013, 8, 1489-1498.
23 J. T. Kwon, H. L. Jiang, A. Minai-Tehrani, C. Gyu Woo, M. Choi, C. S. Cho, Y. S. Kim and M. H. Cho, Iran. J. Pharm. Res., 2013, 12, 281-286.

24 V. Gopal, J. Xavier, G. H. Dar, M. Jafurulla, A. Chattopadhyay and N. M. Rao, Int. J. Pharm., 2011, 419, 347-354.

25 S. Falsini and S. Ristori, Methods Mol. Biol., 2016, 1445, 3343.

26 M. Saunders, K. M. Taylor, D. Q. Craig, K. Palin and H. Robson, Pharm. Res., 2007, 24, 1954-1961.

27 S. Mao, W. Sun and T. Kissel, Adv. Drug Delivery Rev., 2010, 62, 12-27.

28 S. R. Ryoo, H. Jang, K. S. Kim, B. Lee, K. B. Kim, Y. K. Kim, W. S. Yeo, Y. Lee, D. E. Kim and D. H. Min, Biomaterials, 2012, 33, 2754-2761.

29 A. Tahamtan, A. Ghaemi, A. Gorji, H. R. Kalhor, A. Sajadian, A. Tabarraei, A. Moradi, F. Atyabi and M. Kelishadi, J. Biomed. Sci., 2014, 21, 69.

30 Y. K. Kim, M. Zhang, J. J. Lu, F. Xu, B. A. Chen, L. Xing and H. L. Jiang, J. Drug Targeting, 2016, 24, 457-467.

31 J. J. Deng, Y. F. Zhou, B. Xu, K. J. Mai, Y. B. Deng and L. M. Zhang, Biomacromolecules, 2011, 12, 642-649.

32 B. Layek, L. Lipp and J. Singh, J. Controlled Release, 2015, 207, 143-153.

33 E. Baghdan, S. R. Pinnapireddy, B. Strehlow, K. H. Engelhardt, J. Schafer and U. Bakowsky, Int. J. Pharm., 2018, 535, 473-479.

34 X. Huang, X. Huang, X. H. Jiang, F. Q. Hu, Y. Z. Du, Q. F. Zhu and C. S. Jin, J. Microencapsulation, 2012, 29, 1-8.

35 F. Q. Hu, W. W. Chen, M. D. Zhao, H. Yuan and Y. Z. Du, Gene Ther., 2013, 20, 597-606.

36 J. J. Yao, Y. Z. Du, H. Yuan, J. You and F. Q. Hu, Int. J. Nanomed., 2014, 9, 2993-3003.

37 Y. Y. Xu, Y. Z. Du, H. Yuan, L. N. Liu, Y. P. Niu and F. Q. Hu, J. Drug Targeting, 2011, 19, 344-353.

38 J. Yan, Y. Z. Du, F. Y. Chen, J. You, H. Yuan and F. Q. Hu, Mol. Pharmaceutics, 2013, 10, 2568-2577.

39 C. M. Beddoes, C. P. Case and W. H. Briscoe, Adv. Colloid Interface Sci., 2015, 218, 48-68.

40 R. L. Juliano and K. Carver, Adv. Drug Delivery Rev., 2015, 87, 35-45.

41 J. Miao, X. G. Zhang, Y. Hong, Y. F. Rao, Q. Li, X. J. Xie, J. E. Wo and M. W. Li, Carbohydr. Polym., 2012, 87, 13421347.

42 J. J. Zhang, X. Y. Sun, R. Shao, W. Q. Liang, J. Q. Gao and J. L. Chen, J. Drug Delivery Sci. Technol., 2016, 31, 187.

43 M. Saunders, K. M. G. Taylor, D. Q. M. Craig, K. Palin and H. Robson, Pharm. Res., 2007, 24, 1954-1961.

44 S. R. Mao, W. Sun and T. Kissel, Adv. Drug Delivery Rev., 2010, 62, 12-27.

45 Q. Li, Y. Z. Du, H. Yuan, X. G. Zhang, J. Miao, F. D. Cui and F. Q. Hu, Eur. J. Pharm. Sci., 2010, 41, 498-507.

46 Z. X. Du, M. Y. Chen, Q. Q. He, Y. Zhou and T. Jin, Int. J. Pharm., 2012, 434, 437-443. 